\title{
Application analysis of hydraulic nozzle and rotary atomization sprayer on plant protection UAV
}

\author{
J iaqin Gong ${ }^{1 *}$, Wenqing Fan ${ }^{2}$, J in Peng ${ }^{2}$ \\ (XAG Academy, Guangzhou 510663, China)
}

\begin{abstract}
Plant Protection Unmanned Aerial Vehicle(UAV) is the most representative technological product in the construction of precision agriculture in recent years. From direct seeding to areal plant protection, UAV is playing an irreplaceable role. In Japan and China, UAV plant protection has been adopted by more than 4 million farmers in the past 5 years. The sprayer, as an executive component of the plant protection UAV has also made great progress in the past few years. At present, the sprayers being widely used on UAV platforms are Hydraulic Nozzles and Rotary Atomization Sprayers. This article will detail the two popular sprayers and compare them in field applications. The results showed that the rotary atomization sprayer is more suitable for low-volume and variable-rate spraying, both are not possible by hydraulic nozzles. Since variable spraying is an important functionality for precision agriculture, rotary atomization sprayer will be the direction for future UAV and robot sprayer design.
\end{abstract}

Keywords: Plant protection UAV, precision agriculture, rotary atomization sprayer, hydraulic nozzle, variable spraying DOI: $10.33440 /$ j.ijpaa.20190201.0021

Citation: Gong J Q, Fan W Q, Peng J. Application analysis of hydraulic nozzle and rotary atomization sprayer on plant protection UAV. Int J Precis Agric Aviat, 2019; 2(1): 25-29.

\section{Introduction}

China's food crops and cash crops are largely reduced by the erosion of pests and diseases every year. The annual average food loss is nearly 2.5 billion kilograms, accounting for about $20 \%$ of the total output ${ }^{[1]}$. Traditional plant protection methods are manual spraying, tractor spraying, and manned airplane spraying of pesticides. Manual spraying has serious harm to the operator's health as they are exposed to the chemical during application, and the labor efficiency is very low ${ }^{[2]}$. When the pests and diseases occur, it is necessary to spray the pesticides at the fastest speed to control the spread of pests and diseases. It will require a large amount of manpower and material resources to prevent the spread of pests and diseases, which is rather difficult in most areas. When using tractors to spray pesticides, the efficiency is much higher than manual spraying, but because of the size and weight of the machinery, it is restricted by farms geographical conditions. Tractors will also make compaction on the soil and injure the crops during operation. Manned airplane spraying has also been used in many big-farm areas. It requires a big investment for infrastructure such as airports, and flat regular shaped farmlands.

In most of the smallholder farming regions like China and Japan, farmland are usually fragmented, sloped and terraced fields. That is why Large-scale agricultural machinery such as tractors and manned airplanes are not suitable for those countries ${ }^{[3]}$. Moreover,

Received date: 2019-09-16 Accepted date: 2019-10-20

Biographies: Wenqing Fan, Master of Melbourne university, mechanical engineering. Mailing Address: XSpace, 115 Gaopu Rd, Tianhe District. Guangzhou, P.R.C, Email: fanwenqing@xa.com; Jin Peng, Master of South China Agricultural University, UAV Rotary Atomization Sprayers System engineering. Mailing Address: XSpace, 115 Gaopu Rd, Tianhe District. Guangzhou, P.R.C, Email: pengjin@xa.com.

*Corresponding author: Jiaqin Gong, DBA candidate at Université Paris Dauphine and THU, Postgraduate Diploma of Screen Media at Sydney Film School, Master of Professional Accounting at Australia Top Education, Bachelor of Commerce at the University of Sydney. Mailing Address: XSpace, 115 Gaopu Rd, Tianhe District. Guangzhou, P.R.C. Email: justin@xa.com. when using tractors to spread pesticide in later half of the growing season, at least $7 \%$ of the crop will be damaged by the machinery, causing economic losses.

Because of its flexibility and highly automated operation feature, plant protection UAV starts to play an irreplaceable role in the prevention and control of pest and diseases in smallholder economy ${ }^{[4]}$. With the development of areal platforms and spraying systems, plant protection UAV are even capable of spraying large fields economically. Nevertheless, using plant protection UAV to spray can also protect the health of farmers by avoiding direct exposure to chemicals.

Moreover, because the amount of pesticide used in each hectare of land can be accurately calculated, and sprayed by plant protection $\mathrm{UAV}$, it can effectively prevent pesticide residues and environmental pollution caused by excessive use of pesticides.

During the UAV operation, the liquid pesticides will be transformed into small droplets ${ }^{[5]}$ and transported to the crop foliar surface under the action of the sprayer and propeller downwash airflow $^{[6]}$. At the same time, the powerful downwash airflow can cause the plant leaves to flip around, helping the droplets to penetrate the crop canopy thus increase the adhesion rate ${ }^{[7]}$.

Due to the excellent performance of the UAV, it is especially important to equip it with a suitable spray system. At present, the two main types of sprayers being installed on plant protection UAVs are hydraulic nozzles and rotary atomization sprayers. This article will give a detailed introduction and comparison of these two types of sprayers.

\section{Introduction of hydraulic nozzle and rotary atomization sprayer}

Atomization is the process of dispersing a liquid into a more smiling droplet through a nozzle or a high velocity gas stream and ejecting it in a mist. The quality of the nozzle spray is determined by three properties, the uniformity of the droplet distribution, the flowability of the droplet and the coverage of the droplet on the target $6^{[8]}$. These three properties are often contradictory, for 
example, the more concentrated fog shape is beneficial to reduce drift, but the distribution uniformity will be poor and small droplets can increase adhesion but have poor resistance to drift. Therefore, when designing the atomization nozzle, it is necessary to make the trade-offs in three performances.

\subsection{Structure and composition of hydraulic nozzle}

The hydraulic nozzle has a long history and a wide variety of types. The droplets generated by it has a large initial velocity, so the anti-drift ability is very good ${ }^{[9]}$. Nowadays, the hydraulic nozzles for drones are mainly fan hydraulic nozzle. These nozzles are formed by cutting the nozzles on the basis of the real-flow nozzles. The diameter of the nozzle and the angle of the groove are important parameters that affect the droplet size and flowrate ${ }^{[10]}$. The working principle of the hydraulic atomization nozzle is that the liquid is pushed by a hydraulic force through a small opening or an orifice to have sufficient velocity and energy diffusion. First, a liquid film is formed. After that, because the droplets have a high velocity when they leave the nozzle, there is a large speed difference when they come into contact with the outside, so they are split under the action of friction. Thus, the droplets are scattered into unstable, unequal droplets.

Parameter calculation

Hydraulic nozzle related parameters which shows in Figure 1: $h$ is hole margin; $H$ is the groove depth; $e$ is the over-center distance; $D$ is the nozzle hole diameter; $\alpha$ is the groove angle; $\mathrm{Hr}$ is the relative depth of cut ${ }^{[11]}$.

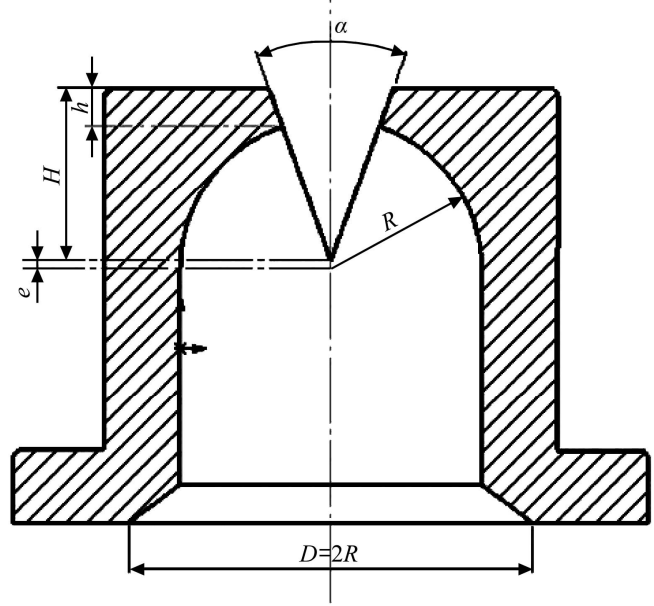

Figure 1 Hydraulic nozzle

According to the principles of fluid mechanics and the Bernoulli equation, the amount of spray $\mathrm{Q}$ is equal to the product of the liquid flow velocity $v$ and the cross-sectional area $\mathrm{F}$ of the nozzle outlet.

$$
Q=\mu \times F=\mu \sqrt{2 g \frac{p}{\gamma}} \times F
$$

where, $g$ is the gravitational acceleration; $\gamma$ is the liquid density; $p$ is the liquid pressure; $\mu$ is the calculated flow rate.

$$
\mu=\frac{1}{\sqrt{1+\sum \mu}}
$$

$\sum \mu$ is the sum of the shrinkage coefficients of the nozzle sections which is determined by the nozzle's own mechanical parameters. Because the actual situation will produce non-linear uncontrollable factors such as flow eddy current, the result of $\mathrm{u}$ calculated by the formula will have a larger error than the actual situation and needs to be corrected by experiments. At present, the calculation of the spray angle has not yet formed a very mature formula, which is mainly calculated on the basis of experiments and calculated by certain fitting and regression methods. According to the derivation, the main parameters affecting the flat fan shape hydraulic nozzle are the nozzle diameter and the groove angle ${ }^{[12]}$.

No matter the diameter of the nozzle and the angle of the groove, once the processing is completed, the parameters cannot be changed, which causes the hydraulic nozzle to not change the droplet size in real time.\#

\subsection{Structure and composition of rotary atomization sprayer}

At present, the main structures of rotary atomization sprayer mounted on plant protection UAV include: liquid cone, motors and atomize disks which shows in Figure 2. The motor shaft is connected to the movement and extends downward from the top of the liquid cone into the bottom of the liquid cone. The atomize disk comprises a turntable and a rotating shaft, and the turntable is located below the liquid cone to receive the liquid discharged from the liquid discharge port. The rotating shaft is fixed at the center of the turntable and extends upward from the liquid discharge opening into the liquid cone to directly connect with the machine shaft. Since the liquid discharge port of the rotary atomization sprayer is sleeved on the rotating shaft, the liquid cone can cast liquid to the center position of the centrifugal spraying disk to effectively improve the spraying uniformity and improve the droplet splashing. Moreover, since the liquid is poured into the atomize disk through the liquid cone, it is not necessary to process the crankshaft into a hollow structure, thereby reducing the processing difficulty and the production $\operatorname{cost}^{[13]}$.

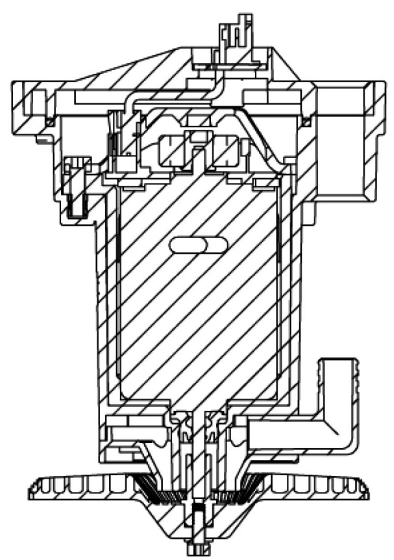

Figure 2 Rotary atomization sprayer

The principle of rotary atomization is that the droplets are lowered on the high-speed optional turntable. The liquid is thrown toward the edge of the turntable by the centrifugal force, and the liquid film is first formed. After being close to the edge, the liquid is split into liquid filaments. After the air hits, it splits again to form a droplet ${ }^{[14]}$. The rotary atomization process includes two forms of atomization, one is centrifugal atomization which is atomized by the gravity of the droplet itself and the centrifugal force, and the centrifugal force plays a major role in this process. The other is that the friction generated by the droplets in contact with the surrounding air causes a finer droplet to be generated, and the friction generated by the speed difference plays a major role in this process.

\section{Comparison}

\subsection{Variable spraying}

The structural principle of the hydraulic nozzle is that the liquid pump pushes the liquid into the nozzle and passively sprays 
the liquid. Therefore, the main advantage of hydraulic nozzle is its large spraying range and high flow rate, and high efficiency spraying to meet the requirements of droplet size. But the principle of hydraulic nozzle made it have a shortcoming which is it cannot come true variable spraying. The only way to change the droplet size is change the nozzle size. Without changing the nozzle, the nozzle system cannot control the spraying effect accurately. Besides that, because the type of nozzle is limited, the optional working state is also limited, and it is difficult to achieve the optimal spraying according to the specific environment. At the same time, because changing spraying parameters must change the characteristics of nozzle, so that hydraulic nozzle cannot adjust in the working process, in the face of sudden changes in wind speed and other environmental changes cannot make timely response, often resulting in waste, over-spraying, or inadequate pesticide adhesion. On the other hand, because the aperture of the hydraulic nozzle is very small, it is prone to blockage, which may cause pipeline rupture, pressure explosion and even damage the UAV platform.

Comparing with the hydraulic nozzle, rotary atomization spray can adjust of spraying parameters in real time without stopping operation, because the droplet size of the rotary atomization sprayer is controlled by the flow rate of the pump and the rotating speed of the rotary disk which can be changed immediately. When facing the change of wind speed and other environmental conditions, the spraying strategy can be adjusted quickly to achieve precise spraying and variable spraying.

In the actual field experiments, it was found that the precise droplet size had an important impact on the spraying effect. When research the control of Lymantria dispar, it was found that: for the 2,3 instar larvae the mortality rate was more than $90 \%$ when the droplet size was 100 micron and the droplet density was 5 and $10 \mathrm{~cm}^{-2}$. For the 4th instar larvae, when the droplet size was 200 and 300 microns, the control effect was more remarkable than that when the droplet size was 100 microns. When the droplet size is $1 \mathrm{~cm}^{-2}$ and the droplet size is 100 , it is ineffective to control 3, 4 instar larvae $\mathrm{e}^{[15]}$.

It can be seen that in practical application, strict control of droplet size is necessary. Because the hydraulic nozzle needs to be replaced when changing the droplet size, and the type of nozzle is limited, the hydraulic nozzle cannot accomplish such spraying task when the droplet size needs to be precisely controlled and constantly changed. On the contrary, rotary atomization spray can provide a much larger variety of droplet sizes than hydraulic nozzles and can be changed in real time.

In addition, in practical application, because the fly speed of UAV during the flight process of spraying operation will change, in order to ensure the uniform spraying quantity, the spraying quantity of nozzle needs to be adjusted according to the fly speed. Take XAG P30 2018 aircraft as an example. When the UAV flies over route in Figure 3.

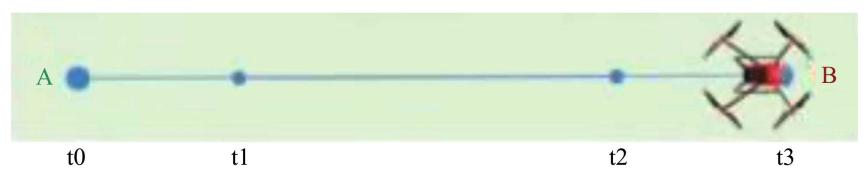

Figure 3 Fly route

Acceleration phase: T01 process, the speed of UAV increases gradually until the speed reaches the target speed (such as $5 \mathrm{~m} / \mathrm{s}$ ).

Uniform speed stage: T1-T2 process, the UAV maintains a uniform speed (e.g. $5 \mathrm{~m} / \mathrm{s}$ ).
Deceleration stage: T2-T3 process, the speed of the UAV gradually decreased, the speed is $0 \mathrm{~m}$ seconds. The relationship between speed, spray volume and time is shown in the Figure 4 and 5 .

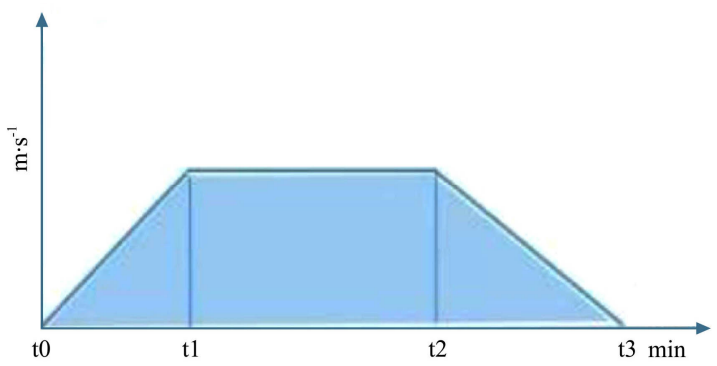

Figure 4 Speed vs time

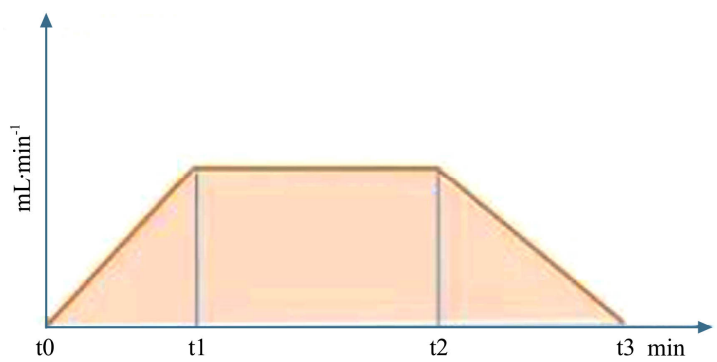

Figure 5 Spray volume vs time

Precision spraying requires uniformity spraying at each place, neither less spraying nor more spraying. It requires that each unit of area spraying quantity remain constant and cannot diverse with the changing of flight speed. It is impossible for UAV to spray at constant spraying velocity.

\subsection{Stability and Reliability property}

It is an important index to measure the nozzle performance that the nozzle can ensure the stable spraying of specific droplet size. In the experiment, the relative span $S$ is a measure of the droplet size distribution $\operatorname{span}^{[16]}$

$$
S=\left(D_{V 90}-D_{V 10}\right) / D_{V 50}
$$

where, $D_{10}$ : Indicates the droplet size with a cumulative volume distribution of $10 \% ; D_{50}$ : The cumulative distribution of droplet volume is $50 \%$ of the droplet size, also known as volume median diameter (VMD); $D_{90}$ : Indicates that the cumulative droplet volume distribution is $90 \%$ of the droplet size.

The smaller the $\mathrm{S}$ value, the more concentrated the distribution of droplet size, the more uniform the spraying particle size. The larger the value of $S$, the more dispersed the distribution of droplet size, the more uneven the droplet size of spraying. For symmetric distribution, $S=1$.

Because the control variable of hydraulic nozzle is only pressure, in order to make the data more credible, three different pressure nozzles are selected to do comparative experiments under three different pressures. The data are as follows ${ }^{[17]}$, showed in Table 1.

Table 1 Hydraulic nozzle experiment results

\begin{tabular}{ccc}
\hline Nozzle type & Pressure/kPa & $S$ \\
\hline \multirow{3}{*}{ TT11002 } & 276 & 1.26 \\
& 300 & 1.14 \\
& 450 & 1.46 \\
\hline \multirow{2}{*}{ XR11002 } & 276 & 1.21 \\
& 300 & 1.33 \\
& 450 & 1.41 \\
\hline \multirow{2}{*}{11001} & 276 & 1.56 \\
& 300 & 1.54 \\
& 450 & 1.45 \\
\hline
\end{tabular}




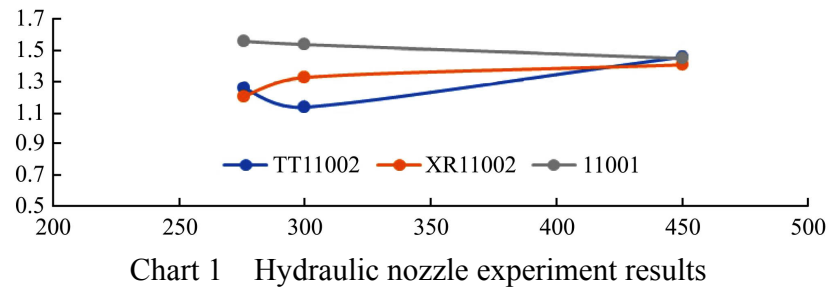

The main parameters of rotary atomization sprayer are spraying amount and motor speed, so two variable parameters are designed in the experiment. The motor speed ranges from $5000 \mathrm{r} / \mathrm{min}$ to $15000 \mathrm{r} / \mathrm{min}$ and the spraying quantity is $350 \mathrm{~mL}$, $450 \mathrm{~mL}$ and $550 \mathrm{~mL}$. The data obtained are showed in Table 2 .

The results show that under various conditions $S$ of hydraulic nozzle is greater than 1 , and $S$ of rotary atomization sprayer is less than 1 , which means the stability property of rotary atomization sprayer is better than hydraulic nozzle.

Table 2 Rotary atomization sprayer experiment results

\begin{tabular}{cccccccccccccc}
\hline Rmp & 5000 & 6000 & 7000 & 8000 & 9000 & 10000 & 11000 & 12000 & 13000 & 14000 & 15000 \\
\hline $350 \mathrm{~mL}$ & 0.77 & 0.80 & 0.78 & 0.75 & 0.73 & 0.71 & 0.72 & 0.72 & 0.71 & 0.70 \\
$450 \mathrm{~mL}$ & 0.71 & 0.77 & 0.76 & 0.75 & 0.78 & 0.76 & 0.81 & 0.73 & 0.73 & 0.75 & 0.75 & 0.74 \\
$550 \mathrm{~mL}$ & 0.88 & 0.79 & 0.77 & 0.81 & 0.72 & 0.82 & 0.80 & 0.80 & 0.79 & 0.78 & 0.76 \\
\hline
\end{tabular}

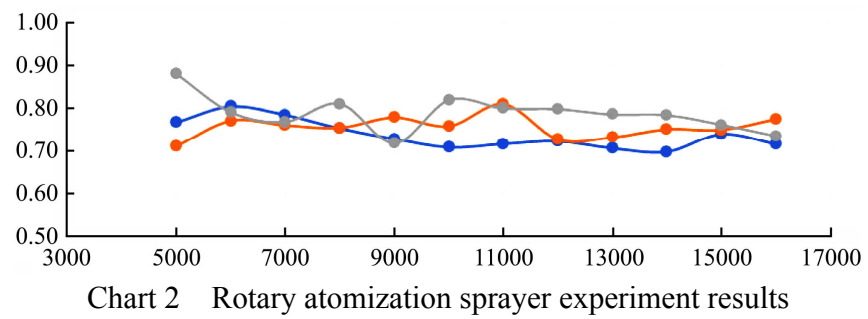

The structure of the hydraulic nozzle determines that it cannot monitor its own spraying state, the spraying effect in the process of operation is not known, and the fault cannot be detected and eliminated in time, which makes the stability and reliability of the hydraulic nozzle not have much room to improve.

In contrast, the principle of the rotary atomization sprayer is to use the peristaltic pump to spray the liquid into the atomize disk. Then the rotary disk drives the liquid atomization and spray it out. The flow rate of spraying liquid is controlled by peristaltic pump, while the droplet size is controlled by rotating speed of rotating disc. Independent and continuous control enables the rotary atomization sprayer to accurately control the spraying effect. At the same time, through the continuous monitoring of the current of the motor on the turntable, the load driven by the turntable and the torque of the motor can be obtained, so that the flow and particle size of the liquid medicine can be accurately detected, and the measured data will be returned to the CPU to control the pump and motor. Such feedback design is an important guarantee for the stability of the system, which further supports the variability and accuracy of spraying.

\section{Conclusions}

The background of the emergence and development of hydraulic nozzle is the promotion of tractor-based mechanized agriculture in the United States at the beginning of the 20th century. Because of its wide spraying range and large flowrate, hydraulic nozzle was very suitable for tractor rapid operation, so it became the preferred scheme at that time. With the development of agricultural technology, UAV has the advantage of low energy consumption and no damage to farmland, and gradually replaces tractor as the best choice of spraying platform. Because of the advantages of high maneuverability and precise positioning of UAV, precise control of spraying can be achieved. This requires that the spraying system can adjust the spraying flow in real time and the particle size to meet the needs of different plants and wind speed. Because the hydraulic nozzle usually cannot meet the optimal spraying requirements and cannot adjust the environmental factors in the operation process, the actual spraying effect cannot achieve precise control, accurately meet the spraying needs and achieve the goal of saving pesticides, water and reducing pollution. Because of the poor stability and high failure rate of the hydraulic nozzle, its competitiveness is further reduced. In contrast, rotary atomization sprayer can adjust spraying effect rapidly according to different wind speed, temperature and plant species. At the same time, spraying state and environmental parameters can be monitored by sensors in real time to achieve accurate spraying, variable spraying, timely changing spraying droplet size when environmental factors such as wind speed suddenly change, resisting the influence of wind speed on adhesion rat, and maximizing the impact to reduce the waste of pesticides, land pollution and achieve maximum spraying effect. In this field, taking Prescription Map as an example, UAV spraying platform with image sensor can accurately analyze the growth of a farmland or even a single plant, and calculate the best spraying strategy. At this time, by changing the working parameters of rotary atomization sprayer, individual plants can be personalized spraying in the best way. Achieve the pursuit of low consumption, low waste and low pollution in precision agriculture. Therefore, rotary atomization sprayer is the best choice for future UAV platform spraying system because it has the advantages of large flow rate and wide spraying range, as well as great advantages in safety, accuracy and working stability.

\section{Research limitation statement}

Most of the nozzles being used on agricultural equipment are hydraulic nozzles, so it is difficult to find a rotary atomization sprayer with good performance designed for plant protection purpose. The sprayers we chose in this research come from XAG Co., Ltd. At present, the application of plant protection UAV covers less than $1 \%$ of world's arable land, mostly in the north and north-west China where crop types are relatively less. This article's research of plant protection UAV spray efficiency is based on broad-acre crops like rice and cotton in Northern China. Moreover, the analysis of spray efficacy is a very complex problem. The droplet size is greatly affected by air temperature and humidity during operation, and the relationship between the factors are nonlinear, so the experiment results are not 100 percent reliable. Different liquids ejected from the same nozzle have different droplet sizes due to the difference of surface tension. In the experiment, we had to replace pesticides with water due to safety concern which may also results in inaccurate results.

Therefore, many improvements can be made in future 
experiments of the sprayers, better droplet size measurement and efficacy evaluation methods are also needed in future studies. At the last, hope this paper has brought some useful information and raised interesting discussion for the other groups which are on similar projects.

\section{[References]}

[1] Liang Lihuang. Analysis on the control technology of rice diseases and insect pests. New Countryside (CN23-1552/S), 2012, 7): 59.(in Chinese)

[2] Fu Zetian, Qi Lijun, Wang Junhong. Research progress and countermeasures of precision application technology. Transactions of the CSAM, 2007, (01): 189-192. (in Chinese)

[3] Zhu Yanzhi. Research on flight control technology of small agricultural unmanned helicopter. Nanjing University of Aeronautics and Astronautics, 2013. (in Chinese)

[4] Huang Y, Hoffmann W C, Lan Y, et al. Development of a spray system for an unmanned aerial vehicle platform. Applied Engineering in Agriculture, 2009, 25(6): 803-809.

[5] Qin W, Xue X, Zhang S, et al. Droplet deposition and efficiency of fungicides sprayed with small UAV against wheat powdery mildew. International Journal of Agricultural and Biological Engineering, 2018, 11(2): 27-32.

[6] Xue Xinyu, Lan Yubin. Analysis on the current situation and development trend of agricultural aviation technology in the united states. Transactions of the CSAM, 2013, 44(05): 194-201.

[7] Chen Shengde, Lan Yubin, F B K, et al. The effect of wind field under the rotor of multi-rotor UAV on the deposition of aviation spray droplets. Transactions of the CSAM, 2017, 48(08): 105-113. (in Chinese)
[8] Fan Qingni. Study on pesticide atomization system of small unmanned helicopter. Nanjing Forestry University, 2011. (in Chinese)

[9] Qi Lijun, Hu Jinrong, Shi Yan, et al. Correlation analysis between spray parameters and drift. Journal of Industrial Engineering, 2004, (05): $122-125$.

[10] Qi Lijun. Study on the optimization of liquid pesticide spraying technology ; China Agricultural University, 2000. (in Chinese)

[11] Wang Lijun, Jiang Minghai, Sun Wenfeng, et al. Selection of Sprinkler Head In Sprayer Design. Journal of Agricultural Mechanization Research, 2005, (02): 151-153. (in Chinese)

[12] Yang Xuejun, Yan Herong, Zhou Haiyan. Experimental study on fan-shaped spray nozzle. Chinese Agricultural Mechanization 2005, (1): 39-42. (in Chinese)

[13] Fan Rong, Shi Shuaibing, Yang Fuzeng, et al. Research status and development trend of common sprayers for plant protection machinery in China. Journal of Agricultural Mechanization Research, 2014, 36(06): 6-9. (in Chinese)

[14] Zhou Lixin, Xue Xinyu, Sun Zhu, et al. Experimental study on electric centrifugal sprinkler for aviation spray. Chinese Agricultural Mechanization, 2011, (01): 107-11. (in Chinese)

[15] Yuan Huizhu, Wang Guobin. The relationship between the droplet size and covering density and the effect of pesticide contro. Plant Protection, 2015, 41(06): 9-16. (in Chinese)

[16] Lan Yubin, Pong Jin, Jin Ji. Research status and development of pesticide spray particle size. Journal of South China Agricultural University, 2016, 37(06): 1-9. (in Chinese)

[17] Zhang Chunhui, Gary D, Zheng Jiaqiang, et al. Wind tunnel test of droplet size distribution of fan-shaped nozzle. Chinese Agricultural Mechanization, 2012, 43(06): 53-57+2. (in Chinese) 